\title{
Zur Entfernung der Nachgeburt nach credé.
}

\author{
Von
}

\section{H. $A$ b e g g}

in Danzig.

Seit Credé 1860 durch seinen Vortrag auf der Naturforscherversammlung in Königsberg sein Verfahren zu allgemeiner Kenntniss brachte, habe ich dasselbe als das zweckmässigste schätzen gelernt, und nicht nur in der Privatpraxis, sondern auch seitdem ich, von $1866 \mathrm{ab}$, die hiesige Hebammenlehranstalt zu leiten habe, in allen geeigneten Fällen, und zwar ohne jeden nachweisbaren Nachtheil, wie ich überzeugt bin, vielmehr zum grössten Nutzen der Gebärenden, angewendet. ${ }^{1}$ )

In der Anstalt geschah von 1866 bis 1880 einsehliesslich die Ausscheidung der Nachgeburt auf folgende Weise:

\begin{tabular}{|c|c|c|c|c|c|c|}
\hline \multirow{2}{*}{$\begin{array}{c}1 . \\
\text { Spontan bin- } \\
\text { nen } 15 \text { Minnten } \\
\text { durch die } \\
\text { Nachgeburts- } \\
\text { wehen allein }\end{array}$} & \multicolumn{4}{|c|}{$\begin{array}{c}2 . \\
\text { Innerlich entfernt: }\end{array}$} & \multirow[b]{2}{*}{ Sa. } & \multirow{2}{*}{$\begin{array}{c}3 . \\
\text { nach Credé. }\end{array}$} \\
\hline & $\begin{array}{c}\text { wegen } \\
\text { schon be- } \\
\text { ginnender } \\
\text { Perimetri- } \\
\text { tis }\end{array}$ & $\begin{array}{l}\text { wegen zu } \\
\text { starken } \\
\text { Fettpol- } \\
\text { sters }\end{array}$ & $\begin{array}{c}\text { wegen } \\
\text { fester Ad- } \\
\text { häsion }\end{array}$ & $\begin{array}{c}\text { wegen zu } \\
\text { tiefen } \\
\text { Sitzes, } \\
\text { Krampf- } \\
\text { wehen }\end{array}$ & & \\
\hline 150 & 6 & 12 & 23 & 182 & 223 & 2993 \\
\hline
\end{tabular}

Hiernach halte ich es für meine Pflicht, da das Verfahren neuerdings von Dohrn, Schultze und Runge wieder bemängelt worden ist, für die entschiedenen Vorzüge desselben einzutreten, namentlich für die Praxis der Hebammen, denen, zumal

1) Abegg, Monatsschrift für Geburtskunde 1861, S. 264. A begg, Zur Geburtshülfe and Gynäkologie. S. 29. Berlin 1868. 
auf dem Lande, doch immer die meisten Geburten allein überlassen bleiben. Die meisten Wochenbetterkrankungen hatten früher nicht die schwere Geburtsarbeit, sondern ungeschickte, rohe Manipulationen der Hebammen bei der inneren Entfernung der Nachgeburt zúr Ursache. Die Besserung, die geringere Häufigkeit der Wochenbettkrankheiten schreibe ich wesentlich dem Credé'schen Verfahren zu.

In Anstalten können die verschiedenen Methoden eher geübt werden, weil gute Aufsicht wenigstens gröbere Missgriffe verhüten wird. Aber auf die Geschicklichkeit, ja auch auf den guten Willen und die Reinlichkeit der ohne ärztliche Aufsicht thätigen Hebammen akann man, wenigstens hier zu Lande, nur selten vertrauen.

Auch in bestgeleiteten Anstalten kommen trotz antiseptischer Cautelen noch Fälle von Sepsis vor, und ungeschickte Hände können, auch wenn sie noch so sorgsam desinficirt sind, bei inneren Eingriffen Unheil genug anrichten.

Deshalb muss gewissenhaften Hebammenlehrern jede Möglichkeit, innere Eingriffe Seitens der Hebammen zu vermeiden, doppelt erwünscht sein. Diese Möglichkeit gewährt aber in Bezug auf die Ausscheidung der Nachgeburt Credé's Verfahren, das leider auch in dem neuesten preussischen Hebammenlehrbuche die verdiente Würdigung nicht gefunden hat. Von gewaltsamen Eingriffen ist bei demselben, wenn es richtig ausgeführt wird, gar keine Rede. - Bei 150 Primiparis wurde ohne jede Reibung des Uterus, nachdem nur als mildester Reiz zu Contractionen einfach eine Hand aufgelegt war, innerhalb einer Viertelstunde durch die Nachgeburtswehen ganz allein die Placenta bis vor oder in die äusseren Geschlechtstheile ausgetrieben. Ist es auch unmöglich, bei den verschiedenen concurrirenden Momenten eine absolute Norm für den Zeitpunkt, die Dauer und die Art der Ausstossung der Nachgeburt zu statuiren, so wird man doch nimmermehr diese spontane, und bald nach der Geburt des Kindes geschehene Entfernung der Placenta als anomal, man wird sie vielmehr als die vorzugsweise normale bezeichnen müssen. Alsdann ist man aber auch berechtigt, diesen Vorgang, wenn er nicht bald nach der Geburt des Kindes eintritt, hervorzurufen, natürlich niemals willkürlich, ausserhalb der Wehen, sondern nur während einer solchen, auch nicht sofort nach der Geburt des Kindes, sondern erst nach wiederholter Contraction des Uterus, in 
der dritten oder vierten Nachgeburtswehe, durch nichts Anderes, als durch Verstärkung derselben.

Es wird gleich nach der Geburt des Kindes eine Hand auf den Uterus gelegt, mit derselben nur dann, wenn in fünf Minuten noch keine Nachgeburtswehe sich einstellt, sanfte Reibungen gemacht, alsdann in der dritten, oder, wenn diese noch schwach ist, aach weiteren Reibungen in der vierten Wehe mit derselben ausgebreiteten Hand der Grund und die Wandungen der Gebärmutter fest umfasst, gleichmässig zusammen und gleichzeitig in der Richtung nach der Kreuzbeinhöhlung nach abwärts gedrückt.

Nachtheile dieses Verfahrens kenne ich nicht. "Dass mitunter, aber nicht oft, Eihautfetzen zurïckbleiben, ist richtig, kommt aber bei innerlicher Herausuahme der Nachgeburt gewiss nicht seltener vor, und ist hier bei der auch von Credé geübten Behandlung, desinficirende Scheideninjectionen und Beförderung der Uteruscontractionen durch Ergotin oder Secale, immer ohne alle erheblichen Zufälle günstig verlaufen. Gewaltsame Versuche, manuell oder gar durch Instrumente die Eihäute aus dem Uterus herauszuholen, können freilich kaum anders als schädlich wirken.

Als bestimmte Gegenanzeigen werden hier betrachtet: schon beginnende Perimetritis, ungewöhnlich starkes Fettpolster und vorher erkannter, zu tiefer Sitz des Mutterkuchens. Bezüglich der Fälle von Krampfwehen bemerke ich noch, dass die meisten in den ersten Jahren sich ereigneten, und, wie ich überzeugt bin, weit weniger zahlreich gewesen wären, wenn ich bei allen hätte zugegen sein können. In dem früheren Lehrbuche war nämlich in erster Linie die innerliche Entfernung der Nachgeburt, und erst dann, wenn diese erfolglos blieb, das Credé'sche Verfahren vorgeschrieben. In meiner Abwesenheit wurde dann danach gehandelt und eben dadurch die Krampfwehen hervorgebracht, durch das damals beliebte Ziehen am Nabelstrange und Reizung des Muttermundes. Sind aber krampfhafte Contractionen des Muttermundes einmal da, so kann vor deren Beseitigung die Credé'sche Methode nicht zur Anwendung kommen.

Mit Credé bin ich der Meinung, dass das Neugeborene, nachdem ihm die Augen ausgewaschen sind und es abgenabelt ist, ohne Schaden auf Baden und Ankleiden warten kann, bis die Nachgeburt entfernt ist. Bis dahin bedarf nicht das Kind, wohl aber die Mutter sorgsamer Beobachtung. 
Ob die Placenta mit der Mitte oder mit einem Rande ihrer Fötalseite voraus aus dem Uterus austritt, ist unwesentlich, wesentlich aber, ob bereits ein grosser Bluterguss stattfand. Ein solcher muss freilich mechanisch die Placenta herabdrängen, schliesslich auch durch starke Ausdehnung des Uterus Contractionen herbeiführen; aber er muss seiner Gefahr wegen möglichst vermieden werden, und er wird vermieden, wenn die Wehen, seien es natürliche oder künstlich erregte, frühzeitig and kräftig eintreten. Denn nur die Wehen, nicht das ergossene Blut, sollen die Placenta hinaustreiben.

Der Druck in der Richtung der Kreuzbeinhöhlung ist, richtig ausgeführt, d. h., wenn die Hohlhand den Muttergrund, die ausgebreiteten Finger aber die Uteruswandungen umfassen, unbedenklich, gerade weil die Befestigungen des Uterus in der Schwangerschaft stark ausgedehnt sind, und durch diesen Handgriff eine noch grössere Ausdehnung nicht erfahren. Wird doch dem Uterus und seinen Befestigungen bei den verschiedensten gynäkologischen Operationen oft genug eine ganz andere Zerrung und Ausdehnung zugemuthet, ohne nachtheilige Folgen!

Mit Credé's') und Fehling's ${ }^{2}$ ) jüngst ausgesprochenen Ansichten muss ich mich daher durchaus einverstanden erklären. Die Einwürfe gegen das Verfahren treffen gar nicht dies selbst, sondern dessen mangelhafte Ausführung.

1) Dieses Archiv, Bd. XVII, Heft 2.

2) Centralblatt für Gynäkologie $1880, \mathrm{Nr} .25$. 